\title{
Hyperviscosity in Non-IgM Lymphoplasmacytic Lymphoma
}

\author{
Mario Fonseca-Paricio, MD, Sushil Ghimire, MD, and Adam Binder, MD
}

\section{CASE}

The following is a case of how a non-IgM paraproteinemia can present with symptoms of hyperviscosity and coagulopathy, an uncommon occurrence. ${ }^{1-3}$ As this case demonstrates, this diagnosis must be considered even in patients with relatively normal coagulation laboratory results. Such pathology requires urgent treatment with plasmapheresis.

SS is a 64-year-old man who presented to the hospital with a chief complaint of fatigue, rash, and leg swelling. He reported feeling significantly more fatigued over the last six months. The fatigue was present throughout the day and marginally improved with rest. He denied any chest pain or dyspnea. He followed with a primary care physician for this fatigue. The workup overall was non-diagnostic. Four weeks prior to presentation to the hospital he developed intermittent night sweats, subjective fevers, and chills. Moreover, for the last month he had noticed swelling in his legs to the level of his knees. Another concerning symptom for SS was a worsening pruritic rash that he first noticed on his lower extremities during the months prior. Initially, the rash was localized, but became more diffuse over the past month.

The patient had no allergies. His only prior medical condition was hypertension for which he was treated with atenolol. There was no family history of malignancy and the patient reported no past or current smoking, alcohol use, or drug use. On review of systems he reported having intermittent black spots on the periphery of his visual fields as well as spontaneous gingival bleeding.

Physical exam revealed diffuse, non-tender, firm lymphadenopathy over the axilla and inguinal regions as well as bilateral lower extremity edema. Skin exam showed diffuse macular erythematous rash with annular formation of palpable purpura over the posterior, superior pelvis, as well as the upper and lower extremities. Laboratory data was notable for hemoglobin of $8.6 \mathrm{~g} / \mathrm{dL}$ with a mean corpuscular volume of $102 \mathrm{fL}$, an elevated protein gap with total protein of $11.2 \mathrm{~g} / \mathrm{dL}$ and albumin of $3.4 \mathrm{~g} / \mathrm{dL}$, as well as an elevated LDH at $790 \mathrm{U} / \mathrm{L}$.

His serum immunoglobulin quantification showed significantly elevated IgG at 5180 mg/dL (723-1,685 mg/ $\mathrm{dL}$ ) with normal IgA and $\mathrm{gM}$ at $75 \mathrm{mg} / \mathrm{dL}(69-382 \mathrm{mg} /$
$\mathrm{dL}$ ) and $140 \mathrm{mg} / \mathrm{dL}(40$ - $230 \mathrm{mg} / \mathrm{dL}$ ) respectively. Serum protein electrophoresis showed a monoclonal protein band in the gamma region at $5.9 \mathrm{~g} / \mathrm{dL}(0.5-1.6 \mathrm{~g} / \mathrm{dL})$, which was further classified as IgG kappa. Kappa/ lambda ratio was calculated at 3.7. His blood repeatedly clotted in the tube despite multiple attempts. Some tests were unable to be performed due to increased serum viscosity. He had inconsistent sodium levels ranging between 113-150 mEq/L without intervention. Hepatic function tests were normal, hepatitis B and C were negative, and cryoglobulin testing was negative. Peripheral flow cytometry showed no abnormal population of B or T cells.

CT of the chest, abdomen, and pelvis showed bilateral axillary, subpectoral, mediastinal, upper abdominal, and retroperitoneal lymphadenopathy with no hepatomegaly or ascites. Skin biopsy of the right thigh showed granular deposition of IgM and C3 in the superficial vessels of the skin consistent with leukocytoclastic vasculitis. Excisional biopsy of the left axillary lymph node showed a small B-cell lymphoma with plasmacytic differentiation. Flow cytometry demonstrated a CD20 +, CD5 -, CD10 -, monoclonal population. CD138 staining showed a separate abundant plasma cell population and kappa light chain restriction. Ki 67 was 10 percent. Bone marrow biopsy showed no evidence of disease. These findings are consistent with lymphoplasmacytic lymphoma (LPL).

His hospital course was complicated by new hematuria during his admission. PT time was slightly prolonged at $14.6 \mathrm{sec}$ with a normal PTT; there were normal levels of coagulation factors II, VII, X, XIII and fibrinogen. Despite not having any laboratory data to confirm hyperviscosity, the intermittent gingival bleeding, blurred vision, and hematuria in the setting of IgG paraproteinemia was consistent with hyperviscosity syndrome; for this, our patient received urgent treatment.

The patient was placed on dexamethasone $40 \mathrm{mg}$ daily for 4 days and received 2 sessions of therapeutic plasma exchange (TPE). After the TPE, his IgG protein decreased to $1,899 \mathrm{mg} / \mathrm{dL}$ with clinical improvement. He was deemed to be medically ready for discharge for outpatient treatment with rituximab and bendamustine for his non-IgM LPL presenting with hyperviscosity syndrome. 


\section{DISCUSSION}

What makes this case rare is not only the diagnosis of a non-IgM LPL, but also the clinical presentation of hyperviscosity syndrome at IgG levels that usually do not result in hyperviscosity. LPL is an indolent disease of mature B cells with plasmacytic differentiation which usually involves bone marrow and less frequently lymph nodes and spleen. ${ }^{1}$ It is responsible for approximately $1 \%$ of all hematological malignancies in the United States. ${ }^{2}$ Among LPLs, the most common form of $L P L$ is $\operatorname{lgM~} L P L$, accounting for approximately $95 \%$ of cases: with non-IgM LPL accounting for approximately $5 \%$ of cases.

The presence of LPL with a monoclonal IgM, the most common subtype, confers the diagnosis of Waldenstrom's macroglobulinemia. ${ }^{3}$ Hyperviscosity is a well-recognized clinical syndrome of Waldenstrom's macroglobulinemia due to increase production of the pentameter immunoglobulin IgM, the largest immunoglobulin with a molecular weight of $990 \mathrm{kDa} .{ }^{4}$ As a result, hyperviscosity can occur at IgM levels as low as 3,000 $\mathrm{mg} / \mathrm{dL}$. This patient's hyperviscosity, instead, presented with an elevated IgG, a smaller immunoglobulin with molecular weight $150 \mathrm{kDa}$. It is thereby less commonly associated with symptoms of hyperviscosity and not typically until levels are significantly elevated. ${ }^{5}$

The levels at which an IgG paraproteinemia would present with hyperviscosity are inconsistent across studies. ${ }^{6,7}$ There is some data that suggests that the risk of hyperviscosity can vary with IgG subclass. For example, IgG3 subclass can produce hyperviscosity at lower levels compared to IgG1, partially explained by the ability of certain subclasses to form concentration dependent aggregates. ${ }^{89}$ This phenomenon was described in a case report in which the low molecular weight IgG molecules aggregated to form trimers and tetramers resulting in larger molecules and clinical hyperviscosity syndrome. ${ }^{10}$

Regardless of the threshold and quantity of immunoglobulins, the American Society for Apheresis defines hyperviscosity syndrome as a category I indication for TPE. ${ }^{11}$ Our patient received 2 sessions of TPE as well as 4 doses of dexamethasone which overlapped with TPE and showed good response to treatment.

\section{CONCLUSION}

LPL has an indolent presentation due to proliferative small B cells with plasma cells or plasmatoid lymphocytes. It is important to stress that in a patient such as ours presenting with signs of a coagulopathy such as visual changes, gingival bleeding, and hematuria in the setting of relatively normal coagulopathy labs to consider hyperviscosity syndrome. It should also be considered in the presence of elevated total protein and widened protein gap. The hyperviscosity could be due to a gammopathy such as the case presented as well as multiple myeloma, acute leukemia, or polycythemias. In the case of hyperviscosity secondary to a gammopathy, the treatment of choice is TPE.

\section{REFERENCES}

1. Swerdlow S, Campo E, Pileri S, et al. The 2016 revision of the World Health Organization classification of lymphoid neoplasms. Blood 2016;127(20):23752390.

2. Morton L, Wang S, Devesa S, et al. Lymphoma incidence patterns by WHO subtype in the United States, 1992-2001. Blood 2006;107(1):265-276.

3. Dimopoulos M, Alexanian R. Waldenstrom's macroglobulinemia. Blood 1994;83(6):1452-1459.

4. Kabat E. The Molecular Weight of Antibodies. Journal of Experimental Medicine 1939:6(1):103-109

5. Mackenzie M, Fudenberg, H, O'Reilly R. The hyperviscosity syndrome in IgG myeloma. The role of protein concentration and molecular shape. The Journal of Clinical Investigation 1970;49(1):15-20.

6. Geha R, Tierney L. Blood Is Thicker: Hyperviscosity Syndrome. The American Journal of Medicine 2018;131(8):916-917

7. Pruzanski W. Serum Viscosity and Hyperviscosity Syndrome in IgG Multiple Myeloma. Annals of Internal Medicine 1972;77(6):853.

8. Virella G. IgG subclasses in relation to viscosity and cryoglobulin syndromes. BMJ 1971;2(5757):322-322.

9. Franey F, Brych $S$, Kolvenbach C, et al. Increased aggregation propensity of IgG2 subclass over IgG1: Role of conformational changes and covalent character in isolated aggregates. Protein Science 2010;19(9):1605-1615.

10. Benninger $G$, Kreps $S$. Aggregation phenomenon in an IgG multiple myeloma resulting in the hyperviscosity syndrome. The American Journal of Medicine 1971:51(2):287-293.

11. Schwartz J, Padmanabhan A, Aqui N, et al. Guidelines on the Use of Therapeutic Apheresis in Clinical Practice-Evidence-Based Approach from the Writing Committee of the American Society for Apheresis: The Seventh Special Issue. Journal of Clinical Apheresis 2016;31(3):149-338 